\title{
La lactancia influyó favorablemente en los niveles de glucemia e insulinoresistencia de pacientes con diagnóstico reciente de diabetes gestacional
}

Breastfeeding influenced favorably glucose and insulin resistance levels in patients with newly diagnosed gestational diabetes mellitus

\section{Objetivos}

Examinar la asociación entre intensidad de la lactancia materna y los niveles de glucemia, insulina y tolerancia a la glucosa en pacientes con antecedente de diabetes gestacional.

\section{Diseño y lugar}

Estudio de cohorte* observacional prospectivo realizado en California, Estados Unidos.

\section{Pacientes}

Se incluyeron 522 mujeres con diabetes gestacional, diagnosticadas entre las 24 y 32 semanas de gestación por medio de una prueba de estimulo con 100 gr. de glucosa según criterios de la Asociación Americana de Diabetes (sigla en inglés, ADA). Además, las pacientes debían presentar una gestación única que finalizara con recién nacidos vivos de 35 o más semanas de edad gestacional.

Evaluación de factores pronósticos y medición de resultados principales

A las seis y nueve semanas post parto se midió glucemia e insulinemia en ayunas y a las dos hs. de administrar $75 \mathrm{gr}$. de glucosa por vía oral (P75). Se determinó asimismo el índice HOMA $^{\S}$ y la sensibilidad a la insulina. Se establecieron cuatro grupos de comparación: lactancia materna exclusiva o predominante (utilización $<180 \mathrm{ml}$. de fórmula en 24hs.) y alimentación exclusiva o predominante con fórmula (utilización $>510 \mathrm{ml}$. de formula en 24hs.). De acuerdo a los valores de glucemia en ayunas y P75 se estableció además el porcentaje de pacientes que reunieron criterios para pre-diabetes (glucemia en ayunas alterada e intolerancia a la glucosa) o diabetes.
Gunderson EP y col. Diabetes Care 2012;35:50-56

\section{Resultados}

Los principales resultados se resumen en la tabla 1. Los grupos de lactancia exclusiva y predominante tuvieron menor prevalencia de diabetes o pre-diabetes $(p=0,02)$.

Tabla 1. Diferencia promedio en los niveles maternos de glucemia, insulinemia e insulinorresistencia de acuerdo al tipo de lactancia a las seis a nueve semanas post parto.

\begin{tabular}{l|c|c}
\multirow{1}{*}{ Resultado } & \multicolumn{2}{|c}{ Diferencia promedio en los grupos (IC95\%)** } \\
\cline { 2 - 3 } & $\begin{array}{l}\text { Lactancia exclusiva vs } \\
\text { alimentación por fórmula } \\
\text { exclusiva o predominante }\end{array}$ & $\begin{array}{c}\text { Lactancia predominante vs } \\
\text { alimentación por fórmula } \\
\text { exclusiva o predominante }\end{array}$ \\
\hline $\begin{array}{l}\text { Glucemia en ayunas } \\
\text { (mg/dl) }\end{array}$ & $-4,3(-7,4$ a $-1,3)$ & $-5,0(-8,5$ a $-1,4)$ \\
\hline Glucemia a las 2 horas & $4,1(-5,0$ a 13,2$)$ & $3,2(27,4$ a 13,8$)$ \\
\hline $\begin{array}{l}\text { Insulinemia en ayunas } \\
\text { ( } \mu \text { U/ml) }\end{array}$ & $-6,3(-10,1$ a $-2,4)$ & $-7,5(-11,9$ a -3$)$ \\
\hline Indice HOMA & $-1,79(-2,86$ a $-0,73)$ & $-2,18(-3,42$ a $-0,95)$ \\
\hline
\end{tabular}

**Ajustado por raza, paridad basal, edad, índice de masa corporal, educación, semana postparto y horas de ayuno previo a las pruebas diagnósticas.

\section{Conclusiones}

Una mayor intensidad de la lactancia se asoció con una mejora de los niveles de glucemia en ayunas y menores niveles de insulina en la evaluación pos-parto (seis a nueve semanas). La lactancia podría tener efectos favorables sobre el metabolismo de la glucosa y la sensibilidad a la insulina, y así reducir el riesgo de diabetes después del embarazo en pacientes con diagnostico previo de diabetes gestacional.

Fuentes de financiamiento: National Institute of Child Health and Human Development, National Institutes of Health National Center for Research Resources.

\section{Comentario}

El valor de identificar a las mujeres con riesgo posterior de desarrollar diabetes está claro. A diferencia de la población incluida en este trabajo, en nuestro medio ésta se integra mayormente por pacientes de origen hispánico. En este último grupo la prevalencia de la diabetes gestacional es mayor y se encuentra entre el 4 y el $10 \%{ }^{1}$. La incidencia de diabetes mellitus postparto, intolerancia a los hidratos de carbono y anormalidades lipídicas es más elevada en las paciente que requirieron insulina durante el embarazo.

La prevalencia de la alimentación a pecho fue reportada en nuestro país por el Ministerio de Salud de la Nación en el $2007^{2}$. Se reportó un valor de lactancia completa (definida como lactancia materna exclusiva o predominante el día ante-

rior a la encuesta) del $61 \%, 53 \%$ y $44 \%$ a los dos, cuatro y seis meses de vida, respectivamente.

\section{Conclusiones del comentador}

Los datos de este artículo suman una razón más para seguir incentivando la lactancia materna. Así, en las pacientes con diagnóstico de diabetes gestacional esta práctica favorecería la regresión de los valores de glucemia e insulina a la normalidad, como así también podría disminuir el número de pacientes que desarrollen pre-diabetes o diabetes tipo II luego del puerperio.

Jessica Campos Flores [ Hospital Materno Infantil Ramón Sarda. jcamposf@intramed.net ]

*Ver glosario

Campos Flores $\mathrm{J}$ La lactancia influyó favorablemente en los niveles de glucemia e insulinoresistencia de pacientes con diagnóstico reciente de diabetes gestacional. Evid Act Práct Ambul. Jul-Set 2012;15(3):98. Comentado de: Gunderson EP, Hedderson M M, Chiang V and col. Lactation Intensity and Postpartum Maternal Glucose Tolerance and Insulin Resistance inWomen With Recent GDM. Diabetes Care 2012;35:50-56. PMID 22011407.

\section{Referencias}

1.Basualdo MN. y col. Guía de Práctica Clínica: Diabetes y embarazo Abril 2010 - Diabetes Gestacional (en línea). Disponible en URL www.Sarda.org.ar/profesionales/guias_y trabajos/guias de practica_clinica/diabetes_y embarazo_abril_2010_-diabetes gestacional (Último acceso: setiembre 2012). 2. Ministerio de Salud de la Nación. Situación de lactancia materna en Argentina (en línea). Dirección Nacional de Maternidad e infancia. 2007. Disponible en URL: http://www.bvspediatria.org.ar/lactancia/situacion2007.pdf (último acceso: septiembre 2012)

${ }^{\S}$ La evaluación del modelo homeostático o índice HOMA (del inglés homoeostasis model assessment) es un método propuesto para cuantificar la resistencia a la insulina definida como la respuesta biológica subnormal (pobre captación del glucosa por parte de las células) en respuesta a la insulina. La ecuación de cálculo contempla en valor de insulinemia, glucemia en ayunas y una constante. Debido a su limitaciones de estandarización y de utilidad práctica esta índice ha caído en desuso y no se recomienda actualmente. 